\title{
Scattered linear sets generated by collineations between pencils of lines
}

\author{
Giorgio Donati · Nicola Durante
}

Received: 10 June 2013 / Accepted: 10 March 2014 / Published online: 25 March 2014

(C) Springer Science+Business Media New York 2014

\begin{abstract}
Let $A$ and $B$ be two points of $\mathrm{PG}\left(2, q^{n}\right)$, and let $\Phi$ be a collineation between the pencils of lines with vertices $A$ and $B$. In this paper, we prove that the set of points of intersection of corresponding lines under $\Phi$ is either the union of a scattered $\operatorname{GF}(q)$ linear set of rank $n+1$ with the line $A B$ or the union of $q-1$ scattered $\operatorname{GF}(q)$-linear sets of rank $n$ with $A$ and $B$. We also determine the intersection configurations of two scattered $\operatorname{GF}(q)$-linear sets of rank $n+1$ of $\operatorname{PG}\left(2, q^{n}\right)$ both meeting the line $A B$ in a $\mathrm{GF}(q)$-linear set of pseudoregulus type with transversal points $A$ and $B$.
\end{abstract}

Keywords Linear sets · Collineations $\cdot$ Subgeometries

Mathematics Subject Classification 51E21 $05 \mathrm{~B} 25$

\section{Introduction}

In [3] and [4], a (degenerate or not) $C_{F}$-set in $\mathrm{PG}\left(2, q^{2}\right)$ is defined as the set of points of intersection of corresponding lines under a collineation with accompanying automorphism $x \mapsto x^{q}$ between two pencils of lines with vertices two distinct points $A$ and $B$ mapping the line $A B$ onto itself or not. Every $C_{F}$-set has $q^{2}+1$ points, and it is of type $(0,1,2, q+1)$ with respect to lines of $\operatorname{PG}\left(2, q^{2}\right)$, and every $(q+1)$-secant line intersects a $C_{F}$-set in a Baer subline (see [3]). Moreover, every $C_{F}$-set is the union of $A$, and $B$ with $q-1$ Baer sublines and it is projectively equivalent to the set of $\mathrm{GF}\left(q^{2}\right)$-rational points of the algebraic curve with equation

\footnotetext{
G. Donati $\cdot$ N. Durante $(\bowtie)$

Dipartimento di Matematica e Applicazioni “Caccioppoli”, Università di Napoli "Federico II", Complesso di Monte S. Angelo - Edificio T, via Cintia, 80126 Napoli, Italy

e-mail: ndurante@unina.it

G. Donati

e-mail: giorgio.donati@unina.it
} 


$$
x_{1} x_{2}^{q}-x_{3}^{q+1}=0
$$

A $C_{F}$-set is also one of the possible intersection configurations of two Hermitian curves of $\mathrm{PG}\left(2, q^{2}\right)$ (see e.g. [6]).

In [4], it is proved that every degenerate $C_{F}$-set is the union of the line $A B$ with a Baer subplane meeting the line $A B$ in a Baer subline and vice versa every Baer subplane can be seen as a subset of a degenerate $C_{F}$-set. Moreover, every degenerate $C_{F}$-set is projectively equivalent to the set of $\mathrm{GF}\left(q^{2}\right)$-rational points of the algebraic curve with equation

$$
x_{3}\left(x_{1} x_{3}^{q-1}-x_{2}^{q}\right)=0
$$

In this paper, we extend the definition of a (degenerate or not) $C_{F}$-set to that of a (degenerate or not) $C_{F}^{m}$-set in any projective plane $\operatorname{PG}\left(2, q^{n}\right)$, with $n \geq 2$, by collineations with accompanying automorphism $x \mapsto x^{q^{m}}$ between two pencils of lines with vertices $A$ and $B$. We will prove that every $C_{F}^{m}$-set of $\operatorname{PG}\left(2, q^{n}\right)$ is the union of $A$ and $B$ with $q-1$ scattered GF $(q)$-linear sets of rank $n$ each of which is a projective geometry $\mathrm{PG}(n-1, q)$ embedded in $\mathrm{PG}\left(2, q^{n}\right)$, and that every degenerate $C_{F}^{m}$-set is the union of the line $A B$ with a scattered $\operatorname{GF}(q)$-linear set of rank $n+1$ meeting the line $A B$ in a $\mathrm{GF}(q)$-linear set of pseudoregulus type with transversal points $A$ and $B$ and vice versa. Furthermore, a degenerate $C_{F}^{m}$-set is also the union of the line $A B$ with an affine geometry $\operatorname{AG}(n, q)$ embedded in $\operatorname{PG}\left(2, q^{n}\right)$. We also calculate the full collineation group stabilizing a (degenerate or not) $C_{F}^{m}$-set of $\operatorname{PG}\left(2, q^{n}\right)$.

In the last section, we determine the intersection configurations of two scattered $\mathrm{GF}(q)$-linear sets of rank $n+1$ of $\operatorname{PG}\left(2, q^{n}\right)$ both meeting the line $A B$ in a GF $(q)$-linear set of pseudoregulus type with transversal points $A$ and $B$.

\section{Preliminary results}

Let $\Omega=\operatorname{PG}\left(r-1, q^{n}\right)=\operatorname{PG}\left(V, \operatorname{GF}\left(q^{n}\right)\right), q=p^{h}, p$ a prime. A set $L$ is said a $\mathrm{GF}(q)$-linear set of $\Omega$ of rank $t$ if it is defined by the non-zero vectors of a $\mathrm{GF}(q)$-vector subspace $U$ of $V$ of dimension $t$, that is

$$
L=L_{U}=\left\{\langle\underline{u}\rangle_{\mathrm{GF}\left(q^{n}\right)}: \underline{u} \in U \backslash\{\underline{0}\}\right\} .
$$

Let $\Lambda=\operatorname{PG}\left(W, \operatorname{GF}\left(q^{n}\right)\right)$ be a subspace of $\Omega$ of dimension $s$, we say that $\Lambda$ has weight $i$ with respect to $L_{U}$ if $\operatorname{dim}_{\mathrm{GF}(q)}(W \cap U)=i$. A $\mathrm{GF}(q)$-linear set $L_{U}$ of $\Omega$ of rank $t$ is said scattered if each point of $L_{U}$ has weight 1 with respect to $L_{U}$. In [15], it is proved that $L_{U}$ is a scattered $\operatorname{GF}(q)$-linear set of rank $t$ if and only if $\left|L_{U}\right|=q^{t-1}+q^{t-2}+\ldots+q+1$, and in [1] it is shown that $t \leq r n / 2$. If $L$ is a scattered linear set of $\mathrm{PG}\left(r-1, q^{n}\right)$ of rank $r n / 2$, then it is called a maximum scattered linear set.

If $\operatorname{dim}_{\mathrm{GF}(q)} U=\operatorname{dim}_{\mathrm{GF}\left(q^{n}\right)} V=r$ and $\langle U\rangle_{\mathrm{GF}\left(q^{n}\right)}=V$, then $L_{U} \cong \mathrm{PG}(U, \mathrm{GF}(q))$ is a subgeometry of $\Omega$. In such a case each point has weight 1 , and hence $\left|L_{U}\right|=$ $q^{r-1}+q^{r-2}+\ldots+q+1$. Let $\Sigma=\operatorname{PG}(t, q)$ be a subgeometry of $\Sigma^{*}=\operatorname{PG}\left(t, q^{n}\right)$ 
and suppose that there exists a $(t-r)$-dimensional subspace $\Omega^{*}$ of $\Sigma^{*}$ disjoint from $\Sigma$. Let $\Omega=\operatorname{PG}\left(r-1, q^{n}\right)$ be an $(r-1)$-dimensional subspace of $\Sigma^{*}$ disjoint from $\Omega^{*}$, and let $\Gamma$ be the projection of $\Sigma$ from $\Omega^{*}$ to $\Omega$ i.e. $\Gamma=\left\{\left\langle\Omega^{*}, x\right\rangle \cap \Omega: x \in \Sigma\right\}$. Let $p_{\Omega^{*}, \Omega}$ be the map from $\Sigma^{*} \backslash \Omega^{*}$ to $\Omega$ defined by $x \mapsto\left\langle\Omega^{*}, x\right\rangle \cap \Omega$. We call $\Omega^{*}$ the center and $\Omega$ the axis of $p_{\Omega^{*}, \Omega}$. In [12], the following characterization of $\mathrm{GF}(q)$-linear sets is given:

Theorem 2.1 If $L$ is a projection of $\mathrm{PG}(t, q)$ into $\Omega=\operatorname{PG}\left(r-1, q^{n}\right)$, then $L$ is a $\mathrm{GF}(q)$-linear set of $\Omega$ of rank $t+1$ and $\langle L\rangle=\Omega$. Conversely, if $L$ is a $\mathrm{GF}(q)$-linear set of $\Omega$ of rank $t+1$ and $\langle L\rangle=\Omega$, then either $L$ is a subgeometry of $\Omega$ or there are $a(t-r)$-dimensional subspace $\Omega^{*}$ of $\Sigma^{*}=\mathrm{PG}\left(t, q^{n}\right)$ disjoint from $\Omega$ and $a$ subgeometry $\Sigma$ of $\Sigma^{*}$ disjoint from $\Omega^{*}$ such that $L=p_{\Omega^{*}, \Omega}(\Sigma)$.

In [13], the authors define a class of maximum scattered GF $(q)$-linear sets of the projective line $\mathrm{PG}\left(1, q^{n}\right)$. Let $P_{1}=\left(y_{1}, y_{2}\right)$ and $P_{2}=\left(z_{1}, z_{2}\right)$ be two distinct points of PG $\left(1, q^{n}\right)$, and let $\tau$ be an automorphism of $\operatorname{GF}\left(q^{n}\right)$ such that $\operatorname{Fix}(\tau)=\{x \in$ $\left.\mathrm{GF}\left(q^{n}\right): x^{\tau}=x\right\}=\mathrm{GF}(q)$. For each $\rho \in \mathrm{GF}\left(q^{n}\right)^{*}=\mathrm{GF}\left(q^{n}\right) \backslash\{0\}$, the set

$$
W_{\rho, \tau}=\left\{\left(\lambda y_{1}+\rho \lambda^{\tau} z_{1}, \lambda y_{2}+\rho \lambda^{\tau} z_{2}\right): \lambda \in \mathrm{GF}\left(q^{n}\right)\right\}
$$

is a $\operatorname{GF}(q)$-vector subspace of $\operatorname{GF}\left(q^{n}\right)^{2}$ of dimension $n$, and $L_{\rho, \tau}=L_{W_{\rho, \tau}}$ is a scattered $\mathrm{GF}(q)$-linear set of rank $n$ called of pseudoregulus type. The points $P_{1}$ and $P_{2}$ are the transversal points of $L_{\rho, \tau}$.

For an element $x$ of $\operatorname{GF}\left(q^{n}\right)$, we denote by $N(x)=x^{\frac{q^{n}-1}{q-1}}$ the norm of $x$ over $\mathrm{GF}(q)$.

Remark 2.2 Let $\mathcal{T}$ be a scattered GF $(q)$-linear set of pseudoregulus type of PG $\left(1, q^{n}\right)$ with transversal points $A^{\prime}=(1,0)$ and $B^{\prime}=(0,1)$. From [13], we have that $\mathcal{T}=\left\{\left(x, \rho x^{q^{m}}\right): x \in \operatorname{GF}\left(q^{n}\right)^{*}\right\}$ for some $\rho \in \operatorname{GF}\left(q^{n}\right)^{*}$ and some $m$ with $(m, n)=1$. Hence, $\mathcal{T}=\{(1, b): N(b)=N(\rho)\}$. If $f$ is a projectivity of $\operatorname{PG}\left(1, q^{n}\right)$ fixing both $A^{\prime}$ and $B^{\prime}$ and stabilizing the set $\mathcal{T}$, then $\left(x_{1}, x_{2}\right)^{f}=\left(a^{q^{m}-1} x_{1}, x_{2}\right)$ for some $a \in \mathrm{GF}\left(q^{n}\right)^{*}$. It follows that the group $G=\left\{f:\left(x_{1}, x_{2}\right) \mapsto\left(c x_{1}, x_{2}\right), N(c)=1\right\}$ has order $\frac{q^{n}-1}{q-1}$ and stabilizes the set $\mathcal{T}$. The orbit of a point $P=\left(1, x_{2}\right)$ of PG $\left(1, q^{n}\right) \backslash\left\{A^{\prime}, B^{\prime}\right\}$, with $N\left(x_{2}\right)=N(\rho)$, under the action of $G$, is the set $\mathcal{T}$. The orbits of $G$ on points of $\mathrm{PG}\left(1, q^{n}\right) \backslash\left\{A^{\prime}, B^{\prime}\right\}$ are the sets $\mathcal{O}_{a}=\{(1, b): N(b)=a\}$ for $a \in \mathrm{GF}(q)^{*}$. So, they are all the possible scattered $\operatorname{GF}(q)$-linear set of pseudoregulus type of $\mathrm{PG}\left(1, q^{n}\right)$ with transversal points $A^{\prime}$ and $B^{\prime}$. They are a partition of $\operatorname{PG}\left(1, q^{n}\right) \backslash\left\{A^{\prime}, B^{\prime}\right\}$. The projectivity

$$
\left(\begin{array}{l}
x_{1}^{\prime} \\
x_{2}^{\prime}
\end{array}\right)=\left(\begin{array}{ll}
1 & 0 \\
0 & t
\end{array}\right)\left(\begin{array}{l}
x_{1} \\
x_{2}
\end{array}\right),
$$

with $N(t)=a$, maps the set $\mathcal{O}_{1}$ onto the set $\mathcal{O}_{a}$. Therefore, all the possible $\operatorname{GF}(q)$ linear sets of pseudoregulus type of $\mathrm{PG}\left(1, q^{n}\right)$ with transversal points $A^{\prime}$ and $B^{\prime}$ are projectively equivalent, independently of $m$ and $\rho$. Since the group $\operatorname{PGL}\left(2, q^{n}\right)$ is 2-transitive on the points of $\operatorname{PG}\left(1, q^{n}\right)$, it follows that all $\operatorname{GF}(q)$-linear sets of pseudoregulus type of $\operatorname{PG}\left(1, q^{n}\right)$ are projectively equivalent. 
A blocking set $\mathcal{B}$ in a projective plane $\mathrm{PG}\left(2, q^{n}\right)$ is a set of points meeting every line of $\mathrm{PG}\left(2, q^{n}\right)$; it is called trivial if it contains a line, and it is called minimal if no proper subset of it is a blocking set. A blocking set $\mathcal{B}$ is small if it has size less than $\frac{3\left(q^{n}+1\right)}{2}$ and it is of Rédei type if there exists a line $\ell$ such that $|\mathcal{B} \backslash \ell|=q^{n}$, the line $\ell$ is called a Rédei line of $\mathcal{B}$. If $\mathcal{B}$ is a $\operatorname{GF}(q)$-linear set of rank $n+1$, then from [16], it follows that $\mathcal{B}$ is a small minimal blocking set called a $\mathrm{GF}(q)$-linear blocking set. Such blocking sets were introduced by G. Lunardon (see [10], [11]).

A linear space is an incidence structure $(\mathcal{P}, \mathcal{L})$, where $\mathcal{P}$ is a nonempty set whose elements are called points, $\mathcal{L}$ is a set, with at least two elements, whose elements are called lines, satisfying the following properties:

- any two distinct points are incident with a unique line;

- every line is incident with at least two distinct points.

Lenz in [9] proved the following characterization theorem for affine spaces in terms of linear spaces:

Theorem 2.3 Let $(\mathcal{P}, \mathcal{L})$ be a linear space with an equivalence relation ("parallelism”) among its lines. If the following properties are satisfied:

(i) Through any point not on a line $\ell$ there is a unique line parallel to $\ell$, and

(ii) Let $\ell$ and $\ell^{\prime}$ be two distinct parallel lines, and let $P, Q$ be two distinct points on $\ell$ and $P^{\prime}$ be a point of $\ell^{\prime}$. If $R$ is a point on $P P^{\prime} \backslash\{P\}$, then the lines $R Q$ and $\ell^{\prime}$ intersect,

then $(\mathcal{P}, \mathcal{L})$ is an affine space.

\section{$3 C_{F}^{m}$-sets in $\operatorname{PG}\left(2, q^{n}\right)$}

Let $A$ and $B$ be two distinct points of a projective plane $\mathrm{PG}\left(2, q^{n}\right)$ over the Galois field $\mathrm{GF}\left(q^{n}\right), q=p^{h}, p$ a prime number, and let $\mathcal{P}_{A}$ and $\mathcal{P}_{B}$ be the pencils of lines with vertices $A$ and $B$, respectively. Let $\alpha_{F}$ be the Frobenius automorphism of $\operatorname{GF}\left(q^{n}\right)$ given by $\alpha_{F}: x \in \mathrm{GF}\left(q^{n}\right) \longrightarrow x^{q^{m}} \in \mathrm{GF}\left(q^{n}\right)$, and let $\Phi$ be an $\alpha_{F}$-collineation between $\mathcal{P}_{A}$ and $\mathcal{P}_{B}$ which does not map the line $A B$ onto itself.

A $C_{F}^{m}-$ set of $\operatorname{PG}\left(2, q^{n}\right)$ is the set of points of intersection of corresponding lines under $\Phi$. The lines $(A B)^{\Phi}$ and $(A B)^{\Phi^{-1}}$ meet at a point $C$ which do not lie on the line $A B$. We may, therefore, take $A, B, C$ as triangle of reference, i.e., $A=(1,0,0)$, $B=(0,1,0)$, and $C=(0,0,1)$.

The collineation $\Phi$ maps the line $a x_{2}+b x_{3}=0$ through $A$ onto the line $\left(\rho a^{q^{m}}+\right.$ $\left.\lambda b^{q^{m}}\right) x_{1}+\left(\mu a^{q^{m}}+\theta b^{q^{m}}\right) x_{3}=0$ through $B$, where $\left(\begin{array}{cc}\rho & \lambda \\ \mu & \theta\end{array}\right)$ is a non-singular matrix over $\operatorname{GF}\left(q^{n}\right)$. Since $\Phi$ maps the line $x_{2}=0$ onto the line $x_{3}=0$ and the line $x_{3}=0$ onto the line $x_{1}=0, \rho$ and $\theta$ are both zero and the set of points of intersection of corresponding lines under $\Phi$ has equation $\lambda x_{1} x_{2} q^{m}-\mu x_{3} q^{m}+1=0$, with $\lambda \mu \neq 0$. Then, via the projectivity

$$
\left(\begin{array}{l}
x_{1}^{\prime} \\
x_{2}^{\prime} \\
x_{3}^{\prime}
\end{array}\right)=\left(\begin{array}{ccc}
\lambda \mu^{-1} & 0 & 0 \\
0 & 1 & 0 \\
0 & 0 & 1
\end{array}\right)\left(\begin{array}{l}
x_{1} \\
x_{2} \\
x_{3}
\end{array}\right),
$$


a $C_{F}^{m}$-set is projectively equivalent to the set of $\mathrm{GF}\left(q^{n}\right)$-rational points of the algebraic curve with equation $x_{1} x_{2} q^{m}-x_{3} q^{m}+1=0$.

Every line through $A$ (respectively through $B$ ) is a 2-secant line except the line $A C$ (respectively the line $B C$ ) which is a tangent line, so every $C_{F}^{m}$-set has $q^{n}+1$ points. The points $A$ and $B$ are called the vertices, and the point $C$, that is the point of intersection of the tangent lines at $A$ and at $B$, is called the center of the $C_{F}^{m}$-set.

Without loss of generality, we may assume $(m, n)=1$ and $m \leq n / 2$. Indeed if $m>n / 2$, then from $x_{1} x_{2} q^{m}-x_{3} q^{m}+1=0$, it follows that $x_{1}^{q^{n-m}} x_{2}-x_{3} q^{n-m}+1=0$. If $m=n / 2$, then from $(m, n)=1$ it follows $m=1$ and $n=2$. Since a $C_{F}^{1}$ set of $\mathrm{PG}\left(2, q^{2}\right)$ has been already studied in [3] (where it was called a $C_{F}$-set) in the sequel, we may suppose that $m<n / 2$.

Note that for $n=m=1$, a $C_{F}^{1}$-set of $\operatorname{PG}(2, q)$ is a non degenerate conic of $\mathrm{PG}(2, q)$ as consequence of Steiner's projective generation of conics (see [17]).

Proposition 3.1 Every line of $\mathrm{PG}\left(2, q^{n}\right)$ intersects a $C_{F}^{m}$-set in $0,1,2$, or $q+1$ points. In the last case, these points form a subline over $\mathrm{GF}(q)$.

Proof Let $\mathcal{C}$ be a $C_{F}^{m}$-set, and let $\ell$ be a line of PG $\left(2, q^{n}\right)$. If $\ell$ contains either $A$ or $B$, then it is either a tangent line or a 2-secant line, so we may suppose that $\ell$ is neither through $A$ nor through $B$. In that case, $\Phi$ induces an $\alpha_{F}$-collineation $\phi_{\ell}$ of the line $\ell$ into itself defined by

$$
\phi_{\ell}: P \in \ell \longrightarrow(A P)^{\Phi} \cap \ell \in \ell .
$$

The points of $\ell \cap \mathcal{C}$ are exactly the fixed points of $\phi_{\ell}$. The system of fixed points of $\phi_{\ell}$ may be one of the following: the empty set, one point, two points, or a subline formed by all the points of $\ell$ coordinatized over the subfield Fix $\left(\alpha_{F}\right)$ (see e.g. [2]). This subfield is $\operatorname{GF}(q)$ since $(m, n)=1$.

Lemma 3.2 Let $\ell=\mathrm{PG}(1, F)$ be a projective line over a field $F$, and let $\sigma$ be an automorphism of $F$. For every subline $\ell^{\prime}$ of the line $\ell$, coordinatized over the subfield Fix $(\sigma)$, there exists only one $\sigma$-collineation of the line $\ell$ into itself fixing pointwise the subline $\ell^{\prime}$.

Proof Let $f$ and $g$ be two $\sigma$-collineations of the line $\ell$ fixing pointwise the subline $\ell^{\prime}$. Then, $f^{-1} \circ g$ is a projectivity of the line $\ell$ fixing pointwise $\ell^{\prime}$. Since $\left|\ell^{\prime}\right| \geq 3$, from the fundamental theorem of projective geometry, we have that $f^{-1} \circ g$ is the identity, so $f=g$. Now, take three distinct points $A^{\prime}, B^{\prime}$, and $C^{\prime}$ on $\ell^{\prime}$. We may suppose that $A^{\prime}=(1,0), B^{\prime}=(0,1)$, and $C^{\prime}=(1,1)$; hence, the $\sigma$-collineation induced by the $\sigma$-semilinear map

$$
\left(\begin{array}{l}
x_{1}{ }^{\prime} \\
x_{2}^{\prime}
\end{array}\right)=\left(\begin{array}{ll}
1 & 0 \\
0 & 1
\end{array}\right)\left(\begin{array}{l}
x_{1}{ }^{\sigma} \\
x_{2}{ }^{\sigma}
\end{array}\right)
$$

fixes pointwise $\ell^{\prime}$. 
Proposition 3.3 Let $\ell_{0}$ be a $\mathrm{GF}(q)$-subline of a line $\ell$ of $\mathrm{PG}\left(2, q^{n}\right)$, and let $A, B$ be two points not on $\ell$ such that the point $A B \cap \ell$ is not on $\ell_{0}$. Then, there exists a unique $C_{F}^{m}$-set with vertices $A$ and $B$ that meets $\ell$ in the subline $\ell_{0}$.

Proof Observe that there is a bijective map $\Psi$ between the set of the $\alpha_{F}$-collineations of the line $\ell$ into itself and the set of the $\alpha_{F}$-collineations between the pencils of lines $\mathcal{P}_{A}$ and $\mathcal{P}_{B}$ with vertices $A$ and $B$. To every $\alpha_{F}$-collineation $f, \Psi$ corresponds the $\alpha_{F}$-collineation $\Psi_{f}$ that maps every line $r \in \mathcal{P}_{A}$ onto the line joining the points $B$ and $(r \cap \ell)^{f}$. By the previous lemma, there exists only one $\alpha_{F}$-collineation $f_{\ell}$ of the line $\ell$ into itself fixing pointwise the subline $\ell_{0}$. Since $f_{\ell}$ does not fix $A B \cap \ell, \Psi_{f_{\ell}}$ does not map the line $A B$ onto itself. Moreover, $\Psi_{f_{\ell}}$ is the unique $\alpha_{F}$-collineation between $\mathcal{P}_{A}$ and $\mathcal{P}_{B}$ such that every point on $\ell_{0}$ is a point of intersection of corresponding lines under $\Psi_{f_{\ell}}$. Hence, the $C_{F}^{m}$-set defined by $\Psi_{f_{\ell}}$ is the unique $C_{F}^{m}$-set of $\operatorname{PG}\left(2, q^{n}\right)$ with vertices $A$ and $B$ that meets $\ell$ exactly in the points of the subline $\ell_{0}$.

Proposition 3.4 Every $C_{F}^{m}$-set of $\mathrm{PG}\left(2, q^{n}\right)$ with vertices $A$ and $B$ is the union of $\{A, B\}$ with $q-1$ pairwise disjoint subsets, each of which is a scattered $\mathrm{GF}(q)$-linear set of rank $n$.

Proof Let $\mathcal{C}$ be the $C_{F}^{m}$-set of $\mathrm{PG}\left(2, q^{n}\right)$ with equation $x_{1} x_{2}^{q^{m}}=x_{3}^{q^{m}+1}$. It is $\mathcal{C}=$ $\left\{\left(1, x^{q^{m}+1}, x^{q^{m}}\right): x \in \mathrm{GF}\left(q^{n}\right)\right\} \cup\{B\}$, and hence $\mathcal{C} \backslash\{A, B\}$ is the union of the sets $\mathcal{C}_{a}=\left\{\left(1, x^{q^{m}+1}, x^{q^{m}}\right): x \in \operatorname{GF}\left(q^{n}\right), N(x)=a\right\}$ for $a \in \operatorname{GF}(q)^{*}$. Let $a$ be an element of $\operatorname{GF}(q)^{*}$. The projectivity

$$
\left(\begin{array}{l}
x_{1}^{\prime} \\
x_{2}^{\prime} \\
x_{3}^{\prime}
\end{array}\right)=\left(\begin{array}{ccc}
1 & 0 & 0 \\
0 & t^{q^{m}+1} & 0 \\
0 & 0 & t^{q^{m}}
\end{array}\right)\left(\begin{array}{l}
x_{1} \\
x_{2} \\
x_{3}
\end{array}\right),
$$

with $N(t)=a$, maps the set $\mathcal{C}_{1}$ onto the set $\mathcal{C}_{a}$. So, the sets $\mathcal{C}_{a}$ for $a \in \operatorname{GF}(q)^{*}$ are pairwise projectively equivalent. Let $N_{1}$ be the set of all elements of $\operatorname{GF}\left(q^{n}\right)$ with unitary norm. Consider the set $S_{m}=\left\{z^{q^{n-m}\left(q^{n-m}-1\right)}: z \in \mathrm{GF}\left(q^{n}\right)^{*}\right\}$. Observe that $S_{m}$ is a multiplicative subgroup of $\operatorname{GF}\left(q^{n}\right)^{*}$, and since $(m, n)=1$ it follows that $\left(q^{n-m}-1, q^{n}-1\right)=q-1$. Therefore, $\left|S_{m}\right|=\left|N_{1}\right|$ and so $S_{m}=N_{1}$. Let $\left(1, x^{q^{m}+1}, x^{q^{m}}\right)$ be an element of $\mathcal{C}_{1}$, there is an element $z \in \operatorname{GF}\left(q^{n}\right)^{*}$ such that $x=z^{q^{n-m}\left(q^{n-m}-1\right)}$. Thus, $\mathcal{C}_{1}=\left\{\left(1, z^{q^{n-m}}\left(q^{n-m}-1\right)\left(q^{m}+1\right), z^{q^{n-m}\left(q^{n-m}-1\right) q^{m}}\right): z \in\right.$ $\left.\operatorname{GF}\left(q^{n}\right)^{*}\right\}$. As $z^{q^{n-m} q^{m}}=z^{q^{n}}=z$, we have that $\mathcal{C}_{1}=\left\{\left(z, z^{q^{2(n-m)}}, z^{q^{n-m}}\right): z \in\right.$ $\left.\operatorname{GF}\left(q^{n}\right)^{*}\right\}$. Since $m<n / 2$, it follows that $\mathcal{C}_{1}=\left\{\left(z, z^{q^{n-2 m}}, z^{q^{n-m}}\right): z \in \operatorname{GF}\left(q^{n}\right)^{*}\right\}$, so $\mathcal{C}_{1}$ is a $\operatorname{GF}(q)$-linear set of size $\frac{q^{n}-1}{q-1}$; hence, it is a rank $n$ scattered $\operatorname{GF}(q)$-linear set.

Let $\mathcal{C}$ be a $C_{F}^{m}$-set of $\operatorname{PG}\left(2, q^{n}\right)$ with vertices $A$ and $B$ and center $C$, generated by an $\alpha_{F}$-collineation $\Phi$ between $\mathcal{P}_{A}$ and $\mathcal{P}_{B}$. Let $\mathcal{T}$ be a scattered linear set of pseudoregulus type of the line $B C$ with transversal points $B$ and $C$. Let $\mathcal{T}^{\prime}$ be the set of lines joining the point $A$ with a point $P$ running in $\mathcal{T}$.

A component of $\mathcal{C}$ is the set of points of intersection of the lines of $\mathcal{T}^{\prime}$ with corresponding lines under $\Phi$. It is easy to see that for a $C_{F}^{m}$-set $\mathcal{C}$ of $\operatorname{PG}\left(2, q^{n}\right)$ with equation 
$x_{1} x_{2}^{q^{m}}-x_{3}^{q^{m}+1}=0$, the $q-1$ components of $\mathcal{C}$ are the sets $\mathcal{C}_{a}$ defined in the proof of the previous proposition. Since $\mathcal{C}$ is the union its $q-1$ components with $\{A, B\}$, it follows easily that every $(q+1)$-secant line to $\mathcal{C}$ is a $(q+1)$-secant line to one of its components.

Remark 3.5 Consider the incidence structure $\left(\mathcal{C}_{1}, \mathcal{L}\right)$, where $\mathcal{L}$ is the set of $(q+1)$ secant lines to $\mathcal{C}_{1}$. Embed $\Omega=\operatorname{PG}\left(2, q^{n}\right)$ in $\Sigma^{*}=\operatorname{PG}\left(n-1, q^{n}\right)$ and let $\Omega^{*}$ be an $(n-4)$-dimensional subspace of $\Sigma^{*}$ disjoint from $\Omega$. Consider a subgeometry $\Sigma=\operatorname{PG}(n-1, q)$ of $\Sigma^{*}$ disjoint from $\Omega^{*}$. From Theorem 2.1, it follows that $\mathcal{C}_{1}$ is obtained via the projection $p_{\Omega^{*}, \Omega}$ from $\Omega^{*}$ onto $\Omega$ of the set $\Sigma$. As $\mathcal{C}_{1}$ is a $\operatorname{GF}(q)$-linear set contained in a set of type $(0,1,2, q+1)$, we have that $\mathcal{C}_{1}$ is of type $(0,1, q+1)$. Via the projection $p_{\Omega^{*}, \Omega}$, it follows that Veblen-Young's axiom holds in $\left(\mathcal{C}_{1}, \mathcal{L}\right)$ since it holds in $\Sigma$. Hence, $\left(\mathcal{C}_{1}, \mathcal{L}\right)$ is a projective geometry $\operatorname{PG}(n-1, q)$ such that every three non-collinear points of $\mathcal{C}_{1}$ are also non-collinear points of $\operatorname{PG}\left(2, q^{n}\right)$.

Proposition 3.6 Let $\mathcal{C}$ be a $C_{F}^{m}$-set, and let $\mathcal{C}^{\prime}$ be a $C_{F}^{m^{\prime}}$-set of $\operatorname{PG}\left(2, q^{n}\right)$. The sets $\mathcal{C}$ and $\mathcal{C}^{\prime}$ are $P \Gamma L$-equivalent if and only if $m^{\prime}=m$.

Proof We can assume that $\mathcal{C}=\left\{\left(1, x^{q^{m}+1}, x^{q^{m}}\right): x \in \mathrm{GF}\left(q^{n}\right)\right\} \cup\{B\}$ and $\mathcal{C}^{\prime}=$ $\left\{\left(1, x^{q^{m^{\prime}}+1}, x^{q^{m^{\prime}}}\right): x \in \mathrm{GF}\left(q^{n}\right)\right\} \cup\{B\}$ and that $m \leq m^{\prime}$. Let $f$ be an element of $P \Gamma L\left(3, q^{n}\right)$ with accompanying automorphism $x \mapsto x^{p^{i}}$ that maps $\mathcal{C}$ to $\mathcal{C}^{\prime}$. The map $f$ fixes the set $\{A, B\}$, since $A$ and $B$ are the unique points of both $\mathcal{C}$ and $\mathcal{C}^{\prime}$ through which all lines but one are 2-secant lines. Indeed, every other point $P$ of $\mathcal{C}$ is contained in a component of $\mathcal{C}$, which is a projective geometry $\operatorname{PG}(n-1, q)$. Moreover, $f$ fixes also the set $\{A C, B C\}$ as these are the unique tangents to both $\mathcal{C}$ and $\mathcal{C}^{\prime}$ through $A$ and $B$, so $f$ fixes $C$.

First, suppose that $f$ fixes both $A$ and $B$. In this case, there exist two elements $a, b \in \mathrm{GF}\left(q^{n}\right)^{*}$ such that such that $\left(x_{1}, x_{2}, x_{3}\right)^{f}=\left(a x_{1}^{p^{i}}, b x_{2}^{p^{i}}, x_{3}^{p^{i}}\right)$. Hence, $\left(1, x^{q^{m}+1}, x^{q^{m}}\right)^{f}=\left(a, b x^{\left(p^{h m}+1\right) p^{i}}, x^{p^{h m+i}}\right)$ belongs to $\mathcal{C}^{\prime}$ for every $x \in \operatorname{GF}\left(q^{n}\right)$. It follows that $a b^{q^{m^{\prime}}} x^{\left(p^{h m}+1\right) p^{h m^{\prime}+i}}=x^{p^{h m+i}\left(p^{h m^{\prime}}+1\right)}$; therefore, $\left(x^{p^{h m}-p^{h m^{\prime}}}\right) p^{p^{i}}=a b^{q^{m^{\prime}}}$ that is $\left(x^{1-p^{h\left(m^{\prime}-m\right)}}\right)^{p^{h m+i}}=a b^{p^{h m^{\prime}}}$ for every $x$ in $\operatorname{GF}\left(q^{n}\right)^{*}$. If $\alpha$ denotes the inverse of the automorphism $x \mapsto x^{p^{h m+i}}$, then $x^{1-p^{h\left(m^{\prime}-m\right)}}=\left(a b^{p^{h m^{\prime}}}\right)^{\alpha}$.

Hence, the polynomial $\left(a b^{p^{h m^{\prime}}}\right)^{\alpha} y^{q^{m^{\prime}-m}-1}-1 \in \mathrm{GF}\left(q^{n}\right)[y]$ is a scalar multiple of the polynomial $y^{q^{n}-1}-1$. Therefore, $\left(a b^{p^{h m^{\prime}}}\right)^{\alpha}=1$ and either $m^{\prime}=m$ or $m^{\prime}=$ $n-m$. The latter case cannot occur since $m, m^{\prime}<n / 2$. Next, assume that $A^{f}=B$. In this case, there exist two elements $a, b \in \mathrm{GF}\left(q^{n}\right)^{*}$ such that $\left(x_{1}, x_{2}, x_{3}\right)^{f}=$ $\left(a x_{2}^{p^{i}}, b x_{1}^{p^{i}}, x_{3}^{p^{i}}\right)$. Therefore, $\left(1, x^{q^{m}+1}, x^{q^{m}}\right)^{f}=\left(a x^{\left(p^{h m}+1\right) p^{i}}, b, x^{p^{h m+i}}\right)$ belongs to $\mathcal{C}^{\prime}$ for every $x \in \mathrm{GF}\left(q^{n}\right)$. It follows that $a b^{q^{m^{\prime}}} x^{p^{i}}-x^{p^{h m+h m^{\prime}+i}}=0$, and hence $a b^{q^{m^{\prime}}}-x^{p^{i}\left(p^{h m+h m^{\prime}}-1\right)}=0$ that is $x^{p^{h m+h m^{\prime}}-1}=\left(a b^{q^{m^{\prime}}}\right)^{p^{h n-i}}$ for every $x \in \operatorname{GF}\left(q^{n}\right)^{*}$.

Hence, the polynomial $y^{p^{h m+h m^{\prime}}-1}-\left(a b^{q^{m^{\prime}}}\right)^{p^{h n-i}} \in \mathrm{GF}\left(q^{n}\right)[y]$ is a scalar multiple of the polynomial $y^{p^{h n}-1}-1$. Therefore, $\left(a b^{q^{m^{\prime}}}\right)^{p^{h n-i}}=1$ and $m+m^{\prime}=n$, a contradiction since $m, m^{\prime}<n / 2$. 
Remark 3.7 Let $f$ be a collineation of $\mathrm{PG}\left(2, q^{n}\right)$ with accompanying automorphism $x \mapsto x^{p^{i}}$ stabilizing the $C_{F}^{m}$-set $\mathcal{C}$ with equation $x_{1} x_{2}^{q^{m}}-x_{3}^{q^{m}+1}=0$. From the proof of previous proposition, we have that $f$ fixes $A, B$, and $C$ pointwise; moreover, there exist two elements $a, b$ of $\operatorname{GF}\left(q^{n}\right)^{*}$ such that $a b^{q^{m}}=1$ and

$$
\left(x_{1}, x_{2}, x_{3}\right)^{f}=\left(a x_{1}^{p^{i}}, b x_{2}^{p^{i}}, x_{3}^{p^{i}}\right) .
$$

Hence, the collineation group $G$ stabilizing $\mathcal{C}$ is the semidirect product of the cyclic linear collineation group $H$ of order $q^{n}-1$ whose elements are the projectivities

$$
\left(x_{1}, x_{2}, x_{3}\right) \mapsto\left(a x_{1}, b x_{2}, x_{3}\right),
$$

where $a b^{q^{m}}=1$, with the cyclic group $K$ of order $n h$ generated by the collineation

$$
\left(x_{1}, x_{2}, x_{3}\right) \mapsto\left(x_{1}^{p}, x_{2}^{p}, x_{3}^{p}\right) .
$$

Remark 3.8 1. Let $\mathcal{C}$ be a $C_{F}^{m}$-set of $\mathrm{PG}\left(2, q^{n}\right)$ with vertices $A$ and $B$ and center $C$, and let $H$ be the full linear collineation group stabilizing $\mathcal{C}$. As previously shown, $H$ is a cyclic group of order $q^{n}-1$. The orbit of a point $P$, not on the edges of the triangle $A B C$, under the group $H$ is the unique $C_{F}^{m}$-set of $\operatorname{PG}\left(2, q^{n}\right)$ with vertices $A$ and $B$ and center $C$ containing $P$. Let $T$ be the unique subgroup of $H$ of order $\frac{q^{n}-1}{q-1}$. The orbit of a point $P$, not on the edges of the triangle $A B C$, under the group $T$ is a projective geometry $\mathrm{PG}(n-1, q)$ embedded in $\mathrm{PG}\left(2, q^{n}\right)$.

2. Kestenband in [7] studies in $\operatorname{PG}\left(2, q^{n}\right)$ the set of $\operatorname{GF}\left(q^{n}\right)$-rational points of the algebraic curve with equation $x_{1}^{q+1}+x_{1} x_{2}^{q}+\tau x_{2} x_{3}^{q}+s x_{3}^{q+1}=0$, where $\tau \neq 0$ and $-s$ is not a $(q+1)^{t h}$ power. He proves that such a set is the union of two distinguished points with $q-1$ sets each of which is a projective geometry $\operatorname{PG}(n-1, q)$ embedded in $\operatorname{PG}\left(2, q^{n}\right)$. We observe that this set is a $C_{F}^{1}$-set of $\operatorname{PG}\left(2, q^{n}\right)$.

\section{Degenerate $C_{F}^{m}$-sets in $\operatorname{PG}\left(2, q^{n}\right)$}

Let $\mathcal{P}_{A}$ and $\mathcal{P}_{B}$ be the pencils of lines with vertices two distinct points $A$ and $B$ of a projective plane $\mathrm{PG}\left(2, q^{n}\right)$ over the Galois field $\mathrm{GF}\left(q^{n}\right), q=p^{h}, p$ a prime number. Let $\Phi$ be an $\alpha_{F}$-collineation between $\mathcal{P}_{A}$ and $\mathcal{P}_{B}$ which maps the line $A B$ onto itself. Without loss of generality, we may assume that $A=(1,0,0)$ and $B=$ $(0,1,0)$. The collineation $\Phi$ maps the line $a x_{2}+b x_{3}=0$ through $A$ onto the line $\left(\rho a^{q^{m}}+\lambda b^{q^{m}}\right) x_{1}+\left(\mu a^{q^{m}}+\theta b^{q^{m}}\right) x_{3}=0$ through $B$, where $\left(\begin{array}{cc}\rho & \lambda \\ \mu & \theta\end{array}\right)$ is a non-singular matrix over $\operatorname{GF}\left(q^{n}\right)$. Since $\Phi$ maps the line $x_{3}=0$ onto itself, it follows that $\lambda=0$. The set $\mathcal{C}$ of points of intersection of corresponding lines under $\Phi$ has equation

$$
x_{3}\left(\rho x_{1} x_{3}^{q^{m}-1}+\mu x_{3}^{q^{m}}-\theta x_{2}^{q^{m}}\right)=0
$$

where $\rho \theta \neq 0$, and it is called a degenerate $C_{F}^{m}$-set of $\operatorname{PG}\left(2, q^{n}\right)$. 
Assuming that the point $C=(0,0,1)$ belongs to $\mathcal{C}$, the previous equation assumes the following form:

$$
x_{3}\left(\rho \theta^{-1} x_{1} x_{3}^{q^{m}-1}-x_{2}^{q^{m}}\right)=0 .
$$

Moreover, every degenerate $C_{F}^{m}$-set of $\mathrm{PG}\left(2, q^{n}\right)$ is projectively equivalent to the set of $\operatorname{GF}\left(q^{n}\right)$-rational points of the algebraic curve with equation

$$
x_{3}\left(x_{1} x_{3}^{q^{m}-1}-x_{2}^{q^{m}}\right)=0
$$

via the projectivity

$$
\left(\begin{array}{l}
x_{1}{ }^{\prime} \\
x_{2}{ }^{\prime} \\
x_{3}{ }^{\prime}
\end{array}\right)=\left(\begin{array}{ccc}
\rho \theta^{-1} & 0 & 0 \\
0 & 1 & 0 \\
0 & 0 & 1
\end{array}\right)\left(\begin{array}{l}
x_{1} \\
x_{2} \\
x_{3}
\end{array}\right) .
$$

Every degenerate $C_{F}^{m}$-set of $\mathrm{PG}\left(2, q^{n}\right)$ has $2 q^{n}+1$ points, $q^{n}+1$ of them are the points of the line $A B$. The points $A$ and $B$ are called the vertices of the degenerate $C_{F}^{m}$-set.

We can assume in the sequel that $m \leq n / 2$. Indeed if $m>n / 2$, then from $x_{1} x_{3}^{q^{m}}-$ $x_{3} x_{2}^{q^{m}}=0$, we have that $x_{1}^{q^{n-m}} x_{3}-x_{3}^{q^{n-m}} x_{2}=0$, and hence via the projectivity

$$
\left(\begin{array}{l}
x_{1}{ }^{\prime} \\
x_{2}{ }^{\prime} \\
x_{3}{ }^{\prime}
\end{array}\right)=\left(\begin{array}{lll}
0 & 1 & 0 \\
1 & 0 & 0 \\
0 & 0 & 1
\end{array}\right)\left(\begin{array}{l}
x_{1} \\
x_{2} \\
x_{3}
\end{array}\right),
$$

we obtain $x_{1}^{\prime} x_{3}^{\prime q^{n-m}}-x_{3}^{\prime} x_{2}^{\prime q^{n-m}}=0$.

If $m=n / 2$, then from $(m, n)=1$, it follows $m=1$ and $n=2$. Since a degenerate $C_{F}^{1}$ set of PG $\left(2, q^{2}\right)$ has been already studied in [4] (where it was called a degenerate $C_{F}$-set) in the sequel, we can assume that $m<n / 2$.

Note that for $n=m=1$, a degenerate $C_{F}^{1}$-set of $\mathrm{PG}(2, q)$ is a degenerate conic of $\mathrm{PG}(2, q)$ as consequence of Steiner's projective generation of conics (see [17]).

Next proposition describes the behavior of a degenerate $C_{F}^{m}$-set with respect to lines of $\mathrm{PG}\left(2, q^{n}\right)$ and can be proved similarly to Proposition 3.1.

Proposition 4.1 Every line of $\mathrm{PG}\left(2, q^{n}\right)$, different from the line $A B$, intersects a degenerate $C_{F}^{m}$-set either in 1, 2 or $q+1$ points. In the last case, these points form a subline over $\mathrm{GF}(q)$.

Proposition 4.2 Let $\ell_{0}$ be a $\mathrm{GF}(q)$-subline of a line $\ell$ of $\mathrm{PG}\left(2, q^{n}\right)$, and let $A, B$ be two points not on $\ell$ such that the point $A B \cap \ell$ is on $\ell_{0}$. Then, there exists only one degenerate $C_{F}^{m}$-set of $\operatorname{PG}\left(2, q^{n}\right)$ with vertices $A$ and $B$ that meets $\ell$ in the subline $\ell_{0}$.

Proof Observe that there is a bijective map $\Psi$ between the set of the $\alpha_{F}$-collineations of the line $\ell$ into itself and the set of the $\alpha_{F}$-collineations between the pencils of lines 
$\mathcal{P}_{A}$ and $\mathcal{P}_{B}$ with vertices $A$ and $B$. To every $\alpha_{F}$-collineation $f, \Psi$ corresponds the $\alpha_{F}$-collineation $\Psi_{f}$ defined by

$$
\Psi_{f}: r \in \mathcal{P}_{A} \longrightarrow(r \cap \ell)^{f} B \in \mathcal{P}_{B} .
$$

From Lemma 3.2, there exists only one $\alpha_{F}$-collineation $f_{0}$ of the line $\ell$ into itself fixing pointwise the subline $\ell_{0}$. Since $f_{0}$ fixes $(A B) \cap \ell, \Psi_{f_{0}}$ maps the line $A B$ onto itself. Moreover, $\Psi_{f_{0}}$ is the unique $\alpha_{F}$-collineation between $\mathcal{P}_{A}$ and $\mathcal{P}_{B}$ such that every point on $\ell_{0}$ is a point of intersection of corresponding lines. Hence, the degenerate $C_{F}^{m}$-set defined by $\Psi_{f_{0}}$ is the unique degenerate $C_{F}^{m}$-set of $\mathrm{PG}\left(2, q^{n}\right)$, with vertices $A$ and $B$, that meets $\ell$ exactly in the points of the subline $\ell_{0}$.

Proposition 4.3 Every degenerate $C_{F}^{m}$-set $\mathcal{C}$ of $\mathrm{PG}\left(2, q^{n}\right)$ with vertices $A$ and $B$ is the union of the line $A B$ with a scattered $\mathrm{GF}(q)$-linear set $\mathcal{S}$ of rank $n+1$, such that $\mathcal{S} \cap A B$ is a $\mathrm{GF}(q)$-linear set of pseudoregulus type with transversal points $A$ and $B$ and vice versa.

Proof Let $\mathcal{C}$ be a degenerate $C_{F}^{m}$-set of $\mathrm{PG}\left(2, q^{n}\right)$ with vertices $A$ and $B$ with equation $x_{3}\left(x_{1} x_{3}^{q^{m}-1}-x_{2}^{q^{m}}\right)=0$, and let $\mathcal{A}=\mathcal{C} \backslash A B$. We first prove that the union of the set $\mathcal{A}$ with the set of the directions of $\mathcal{A}$ on the line $A B$ is a scattered $\operatorname{GF}(q)$-linear set of $\mathrm{PG}\left(2, q^{n}\right)$ of rank $n+1$. Let $X=\left(x^{q^{m}}, x, 1\right)$ and $Y=\left(y^{q^{m}}, y, 1\right)$ be any two distinct points of $\mathcal{A}$. The line $X Y$ meets the line $A B$ in the point $\left((y-x)^{q^{m}}, y-x, 0\right)$, and hence the union of the set $\mathcal{A}$ with its directions on the line $A B$ is given by $\mathcal{S}=\left\{\left(z^{q^{m}}, z, a\right): z \in \operatorname{GF}\left(q^{n}\right), a \in \operatorname{GF}(q),(z, a) \neq(0,0)\right\}$. It is clear that $\mathcal{S}$ is a $\operatorname{GF}(q)$-linear set. Since $\mathcal{S}$ has size $q^{n}+q^{n-1}+\cdots+q+1$, it follows that it is a scattered $\mathrm{GF}(q)$-linear set of $\mathrm{PG}\left(2, q^{n}\right)$ of rank $n+1$. Embed $\Omega=\mathrm{PG}\left(2, q^{n}\right)$ in $\Sigma^{*}=\mathrm{PG}\left(n, q^{n}\right)$, and let $\Omega^{*}$ be an $(n-3)$-dimensional subspace of $\Sigma^{*}$ disjoint from $\Omega$. Consider a subgeometry $\Sigma=\operatorname{PG}(n, q)$ of $\Sigma^{*}$ disjoint from $\Omega^{*}$. Let $H$ be the hyperplane of $\Sigma^{*}$ spanned by $\Omega^{*}$ and the line $A B$. From Theorem 2.1 , it follows that $\mathcal{S}$ (resp. $\mathcal{A}$ ) is obtained via the projection $p_{\Omega^{*}, \Omega}$ from $\Omega^{*}$ onto $\Omega$ of the set $\Sigma$ (resp. $\operatorname{AG}(n, q)=\Sigma \backslash H)$. As $\mathcal{C}$ is a set of type $(1,2, q+1)$, we have that $\mathcal{A}$ is of type $(0,1, q)$. Consider the incidence structure $(\mathcal{A}, \mathcal{L})$, where $\mathcal{L}$ is the set of $q$-secant lines to $\mathcal{A}$. Via the projection $p_{\Omega^{*}, \Omega}$, it follows that the axioms in Theorem 2.3 are satisfied in $(\mathcal{A}, \mathcal{L})$ since they hold in $\Sigma \backslash H$. So, $(\mathcal{A}, \mathcal{L})$ is an affine geometry $\operatorname{AG}(n, q)$. Since $\mathcal{S} \cap A B=\left\{\left(z^{q^{m}}, z, 0\right): z \in \operatorname{GF}\left(q^{n}\right)^{*}\right\}$ from Remark 4.7 in [13] it follows that $\mathcal{S} \cap A B$ is a $\mathrm{GF}(q)$-linear set of pseudoregulus type with transversal points $A$ and $B$. Vice versa let $\mathcal{C}$ be the union of the line $A B$ with a scattered $\operatorname{GF}(q)$-linear set $\mathcal{S}$ of rank $n+1$, such that $\mathcal{S} \cap A B$ is a $\operatorname{GF}(q)$-linear set of pseudoregulus type with transversal points $A$ and $B$. We may assume that $\mathcal{S} \cap A B=\left\{\left(z^{q^{m}}, z, 0\right): z \in \operatorname{GF}\left(q^{n}\right)^{*}\right\}$ for some $m \in\{1, \ldots, n-1\}$ with $(m, n)=1$ (see [13]), and from the first part of this proof, the set $\mathcal{S}$ is the projection of $\Sigma$ from $\Omega^{*}$ to $\Omega$. Let $\Psi$ be the collineation of $\Sigma^{*}$ of order $n$ with accompanying automorphism $x \mapsto x^{q}$ such that $\Sigma=F i x(\Psi)$. As in [13], we may assume that $\Omega^{*}=\left\langle B^{\Psi^{i}}: i \neq 0, m\right\rangle$, where $B$ and $A=B^{\Psi^{m}}$ are the transversal points of $\mathcal{S} \cap A B$. Consider the following collineation with accompanying automorphism $x \mapsto x^{q^{m}}$ :

$$
\Phi: \ell \in \mathcal{P}_{B} \rightarrow\left\langle\ell, \Omega^{*}\right\rangle^{\Psi^{m}} \cap \Omega \in \mathcal{P}_{A} .
$$


Since the collineation $\Psi$ fixes the hyperplane $\left\langle\Omega^{*}, A B\right\rangle$, the collineation $\Phi$ maps the line $A B$ into itself. For every line $\ell \in \mathcal{P}_{B}$ with $\ell$ different from $A B$, the hyperplane $\left\langle\ell, \Omega^{*}\right\rangle$ meets $\Sigma$ in a unique point $P$ that is also the unique point of $\Sigma$ in $\left\langle\ell^{\Phi}, \Omega^{*}\right\rangle$, and hence $\ell \cap \ell^{\Phi}$ is the projection of $P$ form $\Omega^{*}$ to $\Omega$. It follows that $\mathcal{C}$ is a degenerate $C_{F}^{m}$-set of $\Omega$.

Remark 4.4 1. From the proof of the previous proposition, it follows that every degenerate $C_{F}^{m}$-set of $\operatorname{PG}\left(2, q^{n}\right)$ is the union of the line $A B$ with a set $\mathcal{A}$ of $q^{n}$ points isomorphic to an affine geometry $\operatorname{AG}(n, q)$ such that every three noncollinear points of $\mathcal{A}$ are also non-collinear points of $\mathrm{PG}\left(2, q^{n}\right)$.

2. The set $\mathcal{S}$ defined in the proof of Proposition 4.3 is a small minimal $\mathrm{GF}(q)$-linear blocking set of Rédei type.

Proposition 4.5 Let $\mathcal{C}$ be a degenerate $C_{F}^{m}$-set, and let $\mathcal{C}^{\prime}$ be a degenerate $C_{F}^{m^{\prime}}$ set of $\operatorname{PG}\left(2, q^{n}\right)\left(m, m^{\prime}<n / 2\right)$. The sets $\mathcal{C}$ and $\mathcal{C}^{\prime}$ are $P \Gamma L$-equivalent if and only if $m^{\prime}=m$.

Proof We can assume that $\mathcal{C}=\left\{\left(x^{q^{m}}, x, 1\right): x \in \mathrm{GF}\left(q^{n}\right)\right\} \cup A B$ and $\mathcal{C}^{\prime}=$ $\left\{\left(x^{q^{m^{\prime}}}, x, 1\right): x \in \operatorname{GF}\left(q^{n}\right)\right\} \cup A B$ and that $m \leq m^{\prime}$. Let $f$ be an element of $P \Gamma L\left(3, q^{n}\right)$ with accompanying automorphism $x \mapsto x^{p^{i}}$ that maps $\mathcal{C}$ in $\mathcal{C}^{\prime}$. From Proposition 4.3, we have that $\mathcal{C}$ (resp. $\mathcal{C}^{\prime}$ ) is the union of the line $A B$ with a scattered $\operatorname{GF}(q)$-linear set $\mathcal{S}$ (resp. $\mathcal{S}^{\prime}$ ), such that $\mathcal{S} \cap A B\left(\mathcal{S}^{\prime} \cap A B\right)$ is a $\mathrm{GF}(q)$-linear set of pseudoregulus type with transversal points $A$ and $B$. The map $f$ sends $\mathcal{S} \cap A B$ to $\mathcal{S}^{\prime} \cap A B$, and these $\mathrm{GF}(q)$-linear sets have the same transversal points. From Proposition 4.3 in [13], the transversal points of a scattered $\operatorname{GF}(q)$-linear set of pseudoregulus type are uniquely determined, so the map $f$ necessarily stabilizes the set $\{A, B\}$.

First, suppose that $f$ fixes both $A$ and $B$. In this case, there exist four elements $a, b, c, d \in \mathrm{GF}\left(q^{n}\right), a c \neq 0$ such that $\left(x_{1}, x_{2}, x_{3}\right)^{f}=\left(a x_{1}^{p^{i}}+b x_{3}^{p^{i}}, c x_{2}^{p^{i}}+d x_{3}^{p^{i}}, x_{3}^{p^{i}}\right)$. Hence, $\left(x^{q^{m}}, x, 1\right)^{f}=\left(a x p^{p^{h m+i}}+b, c x^{p^{i}}+d, 1\right)$ belongs to $\mathcal{C}^{\prime}$ for every $x \in \operatorname{GF}\left(q^{n}\right)$. It follows that $c^{q^{m^{\prime}}} x^{p^{h m^{\prime}+i}}-a x^{p^{h m+i}}+d^{p^{h m^{\prime}}}-b=0$ for every $x \in \operatorname{GF}\left(q^{n}\right)$. As 0 is a solution of the previous equation, we have that $d^{p^{h m^{\prime}}}=b$; thus, $x^{p^{h m+i}}\left(c^{p^{h m^{\prime}}} x^{p^{h m+i}\left(p^{h\left(m^{\prime}-m\right)}-1\right)}-a\right)=0$ for every $x \in \mathrm{GF}\left(q^{n}\right)$. Hence, the polynomial $c^{p^{h m^{\prime}}} y^{p^{h m+i}\left(p^{h\left(m^{\prime}-m\right)}-1\right)}-a \in \mathrm{GF}\left(q^{n}\right)[y]$ is a scalar multiple of the polynomial $y^{p^{h n}-1}-1$. So $m^{\prime}=m$ and $a=c^{p^{h m^{\prime}}}$.

Next, assume that $A^{f}=B$. In this case, there exist four elements $a, b, c, d \in$ $\mathrm{GF}\left(q^{n}\right), a c \neq 0$ such that $\left(x_{1}, x_{2}, x_{3}\right)^{f}=\left(a x_{2}^{p^{i}}+b x_{3}^{p^{i}}, c x_{1}^{p^{i}}+d x_{3}^{p^{i}}, x_{3}^{p^{i}}\right)$. Hence, $\left(x^{q^{m}}, x, 1\right)^{f}=\left(a x p^{p^{i}}+b, c x^{p^{h m+i}}+d, 1\right)$ belongs to $\mathcal{C}^{\prime}$ for every $x \in \operatorname{GF}\left(q^{n}\right)$. It follows that $c^{q^{m^{\prime}}} x^{p^{h m+h m^{\prime}+i}}-a x^{p^{i}}+d^{q^{m^{\prime}}}-b=0$ for every $x \in \operatorname{GF}\left(q^{n}\right)$. As before $d^{q^{m^{\prime}}}=b \operatorname{soc} c^{q^{m^{\prime}}} x^{p^{i}\left(p^{h m+h m^{\prime}}-1\right)}-a=0$ for every $x \in \mathrm{GF}\left(q^{n}\right)^{*}$. Hence, the polynomial $c^{q^{m^{\prime}}}\left(y^{p^{i}}\right)^{p^{h m+h m^{\prime}}-1}-a \in \mathrm{GF}\left(q^{n}\right)[y]$ is a scalar multiple of the polynomial $y^{p^{h n}-1}-1$. Therefore, $m+m^{\prime}=n, i=0, a=c^{q^{m^{\prime}}}$, a contradiction since $m, m^{\prime}<n / 2$.

Remark 4.6 Let $f$ be a collineation of $\mathrm{PG}\left(2, q^{n}\right)$ with accompanying automorphism $x \mapsto x^{p^{i}}$ stabilizing the degenerate $C_{F}^{m}$-set with equation $x_{3}\left(x_{1} x_{3}^{q^{m-1}}-x_{2}^{q^{m}}\right)=0$. 
From the proof of previous proposition, we have that $f$ fixes $\{A, B\}$ pointwise, and that there exist four elements $a, b, c, d$ of $\mathrm{GF}\left(q^{n}\right)$ with $a c \neq 0, a=c^{q^{m}}, b=d^{q^{m}}$ such that

$$
\left(x_{1}, x_{2}, x_{3}\right)^{f}=\left(a x_{1}^{p^{i}}+b x_{3}^{p^{i}}, c x_{2}^{p^{i}}+d x_{3}^{p^{i}}, x_{3}^{p^{i}}\right) .
$$

Hence, the full collineation group stabilizing a degenerate $C_{F}^{m}$-set of $\mathrm{PG}\left(2, q^{n}\right)$ is the semidirect product of the linear collineation group of order $q^{n}\left(q^{n}-1\right)$ whose elements are the projectivities

$$
\left(x_{1}, x_{2}, x_{3}\right) \mapsto\left(a x_{1}+b x_{3}, c x_{2}+d x_{3}, x_{3}\right),
$$

where $a c \neq 0, a=c^{q^{m}}$, and $b=d^{q^{m}}$ with the cyclic group of order $n h$ generated by the collineation

$$
\left(x_{1}, x_{2}, x_{3}\right) \mapsto\left(x_{1}^{p}, x_{2}^{p}, x_{3}^{p}\right)
$$

Remark 4.7 1. Let $\mathcal{C}$ be a degenerate $C_{F}^{m}$-set of $\mathrm{PG}\left(2, q^{n}\right)$, and let $T$ be the linear collineation group of order $q^{n}$, stabilizing $\mathcal{C}$, whose elements are the projectivities $\left(x_{1}, x_{2}, x_{3}\right) \mapsto\left(x_{1}+a^{q^{m}} x_{3}, x_{2}+a x_{3}, x_{3}\right)$ for any $a \in \operatorname{GF}\left(q^{n}\right)$. The orbit of a point $P$, not on the line $A B$, under the action of $T$ is a affine geometry $\operatorname{AG}(n, q)$ embedded in $\mathrm{PG}\left(2, q^{n}\right)$.

2. Let $G$ be the full collineation group stabilizing a degenerate $C_{F}^{m}$-set $\mathcal{C}$ of $\mathrm{PG}\left(2, q^{n}\right)$ calculated in Remark 4.6. Every element of $G$ stabilizes the scattered $\operatorname{GF}(q)$-linear set $\mathcal{S}$, contained in $\mathcal{C}$, of rank $n+1$ of $\mathrm{PG}\left(2, q^{n}\right)$ such that $\mathcal{S} \cap A B$ is a $\operatorname{GF}(q)$ linear set of pseudoregulus type and vice versa. Thus, the full collineation group stabilizing $\mathcal{S}$ coincides with $G$.

\section{Intersection of two scattered GF $(q)$-linear sets of rank $n+1$ in $\operatorname{PG}\left(2, q^{n}\right)$}

In this section, we study the possible intersection configurations of a degenerate $C_{F}^{m}$ set, say $\mathcal{C}$, with a degenerate $C_{F}^{m^{\prime}}$-set, say $\mathcal{C}^{\prime}$, both of $\mathrm{PG}\left(2, q^{n}\right)$ without the restriction $(n, m)=\left(n, m^{\prime}\right)=1$ and with $m \geq m^{\prime}$. We assume that both $\mathcal{C}$ and $\mathcal{C}^{\prime}$ have the same vertices $A$ and $B$. In the meantime, we also study the possible intersection configurations of two scattered $\mathrm{GF}(q)$-linear sets of rank $n+1$ such that both meet $A B$ in a $\mathrm{GF}(q)$-linear set of pseudoregulus type with transversal points $A$ and $B$.

Proposition 5.1 Let $\mathcal{C}$ be a degenerate $C_{F}^{m}$-set of $\mathrm{PG}\left(2, q^{n}\right)$ defined by the collineation $\Phi$, and let $\mathcal{C}^{\prime}$ be a degenerate $C_{F}^{m^{\prime}}$-set of $\mathrm{PG}\left(2, q^{n}\right)$ defined by the collineation $\Phi^{\prime}$. If both $\mathcal{C}$ and $\mathcal{C}^{\prime}$ have the same vertices $A$ and $B$ and $\mathcal{C} \neq \mathcal{C}^{\prime}$, then $\mathcal{C} \cap \mathcal{C}^{\prime} \backslash A B$ is one of the following:

i) the empty set;

ii) one point;

iii) a degenerate $C_{F}^{n-m+m^{\prime}}$-set of a subgeometry $\mathrm{PG}\left(2, q^{t}\right)$ of $\mathrm{PG}\left(2, q^{n}\right)$ minus the line $A B$, where $t=\left(n, n-m+m^{\prime}\right)\left(m \neq m^{\prime}\right)$. 
Proof If $P$ is a point of $\mathcal{C} \cap \mathcal{C}^{\prime} \backslash A B$, then $(A P)^{\Phi}=(A P)^{\Phi^{\prime}}$; hence, the points of $\mathcal{C} \cap \mathcal{C}^{\prime} \backslash A B$ are in one-to-one correspondence with the lines of $\mathcal{P}_{A} \backslash\{A B\}$ fixed by the collineation $\Phi^{-1} \circ \Phi^{\prime}$ with accompanying automorphism $\tau: x \mapsto x^{q^{n-m+m^{\prime}}}$. The set of fixed lines of $\Phi^{-1} \circ \Phi^{\prime}$, different from $A B$, is one of the following: the empty set, a line, and a subpencil of $\mathcal{P}_{A}$ coordinatized over the subfield Fix $(\tau)=\operatorname{GF}\left(q^{t}\right)$, where $t=\left(n, n-m+m^{\prime}\right)$. In this last case by considering $\Phi$ and $\Phi^{\prime}$ restricted to the subpencil Fix $\left(\Phi^{-1} \circ \Phi^{\prime}\right)$, we have that $\mathcal{C} \cap \mathcal{C}^{\prime}$ is a degenerate $C_{F}^{n-m+m^{\prime}}$-set of a subgeometry $\mathrm{PG}\left(2, q^{t}\right)$ of $\mathrm{PG}\left(2, q^{n}\right)$. Observe that if $m=m^{\prime}$, then $\tau$ is the identity, $\Phi^{-1} \circ \Phi^{\prime}$ is a projectivity and so case $\left.i i i\right)$ cannot occur.

Next, we study the intersections of two scattered GF $(q)$-linear sets. We note that the intersection problem for two $\mathrm{GF}(q)$-linear sets generalizes the intersection problem for two subgeometries. Very little is known on the intersection of two $\operatorname{GF}(q)$-linear sets (see [8],[14]), while the intersection problem for two subgeometries was solved in [5].

Proposition 5.2 Let $\mathcal{S}$ and $\mathcal{S}^{\prime}$ be two scattered $\mathrm{GF}(q)$-linear sets of rank $n+1$ of $\mathrm{PG}\left(2, q^{n}\right)$ such that both meet the line $A B$ in a $\mathrm{GF}(q)$-linear set of pseudoregulus type with transversal points $A$ and $B$. Then, $\mathcal{S} \cap \mathcal{S}^{\prime}$ is one of the following:

- the empty set;

- one point;

- a degenerate $C_{F}^{n-m+m^{\prime}}$-set of a subgeometry $\mathrm{PG}\left(2, q^{t}\right)$ of $\mathrm{PG}\left(2, q^{n}\right)$ minus the line $A B$, where $t=\left(n, n-m+m^{\prime}\right)\left(m \neq m^{\prime}\right)$;

- the $\operatorname{GF}(q)$-linear set of pseudoregulus type $\mathcal{S} \cap A B$ with transversal points $A$ and $B$;

- the union of a point with the set $\mathcal{S} \cap A B$;

- the union of a degenerate $C_{F}^{n-m+m^{\prime}}$-set of a subgeometry $\mathrm{PG}\left(2, q^{t}\right)$ of $\mathrm{PG}\left(2, q^{n}\right)$ minus the line $A B$, where $t=\left(n, n-m+m^{\prime}\right)\left(m \neq m^{\prime}\right)$, with the set $\mathcal{S} \cap A B$.

Proof From Proposition 4.3, the union of $\mathcal{S}$ with the line $A B$ is a degenerate $C_{F}^{m}$-set $\mathcal{C}$ of $\operatorname{PG}\left(2, q^{n}\right)$ for some $m$ with $(m, n)=1$, and the union of $\mathcal{S}^{\prime}$ with the line $A B$ is a degenerate $C_{F}^{m^{\prime}}$-set $\mathcal{C}^{\prime}$ of $\mathrm{PG}\left(2, q^{n}\right)$ for some $m^{\prime}$ with $\left(m^{\prime}, n\right)=1$. The possible intersection configurations for $\mathcal{C} \cap \mathcal{C}^{\prime} \backslash A B$ are listed in Proposition 5.1. Moreover, from Remark 2.2, the set $\mathcal{S} \cap \mathcal{S}^{\prime} \cap A B$ is either the empty set or it coincides with $\mathcal{S} \cap A B . \square$

Acknowledgments The authors thank Olga Polverino for her helpful suggestions regarding Proposition 3.4 .

\section{References}

1. Blokhuis, A., Lavrauw, M.: Scattered spaces with respect to a spread in $\mathrm{PG}(n, q)$. Geom. Dedicata 81(1-3), 231-243 (2000)

2. Donati, G.: On the system of fixed points of a collineation in non commutative projective geometry. Discrete Math. 255, 65-70 (2002)

3. Donati, G., Durante, N.: Some subsets of the Hermitian curve. Eur. J. Combin. 24, 211-218 (2003)

4. Donati, G., Durante, N.: Baer subplanes generated by collineations between pencils of lines. Rend. Circ. Mat. Palermo 54(1), 93-100 (2005) 
5. Donati, G., Durante, N.: On the intersection of two subgeometries of PG $(n, q)$. Des. Codes, Cryptogr. 46, 261-267 (2008)

6. Donati, G., Durante, N.: On the intersection of Hermitian curves and of Hermitian surfaces. Discrete Math. 308, 5196-5203 (2008)

7. Kestenband, C.: Embedding finite projective geometries into finite projective planes. Int. Electr. J. Geomet. 2(2), 27-33 (2009)

8. Lavrauw, M., Van de Voorde, G.: On linear sets on a projective line. Des. Codes Cryptogr. 56, 89-104 (2010)

9. Lenz, H.: Zur Begrundung der affinen Geometrie des Raumes. Mitt. Math. Ges. Hamburg 11(6), 763775 (1989)

10. Lunardon, G.: Normal spreads. Geom. Dedicata 75, 245-261 (1999)

11. Lunardon, G.: Linear $k$-blocking sets. Combinatorica 21(4), 571-581 (2001)

12. Lunardon, G., Polverino, O.: Translation Ovoids of Orthogonal Polar Spaces. Forum. Math. 16, 663669 (2004)

13. Lunardon, G., Marino, G., Polverino, O., Trombetti, R.: Maximum scattered linear sets of pseudoregulus type and the Segre Variety $\mathcal{S}_{n, n}$. J. Algebr. Comb. (2013). doi:10.1007/s10801-013-0468-3

14. Pepe, V.: On the algebraic variety $\mathcal{V}_{r, t}$. Finite Fields Appl. 17(4), 343-349 (2011)

15. Polverino, O.: Linear sets in finite projective spaces. Discrete Math. 310, 3096-3107 (2010)

16. Polito, P., Polverino, O.: On small blocking sets. Combinatorica 18(1), 133-137 (1998)

17. Steiner, J.: Systematische Entwichlung der Abh $\ddot{a}$ ngigkeit Geometrische Gestalten von einander. Reimer, Berlin (1832) 